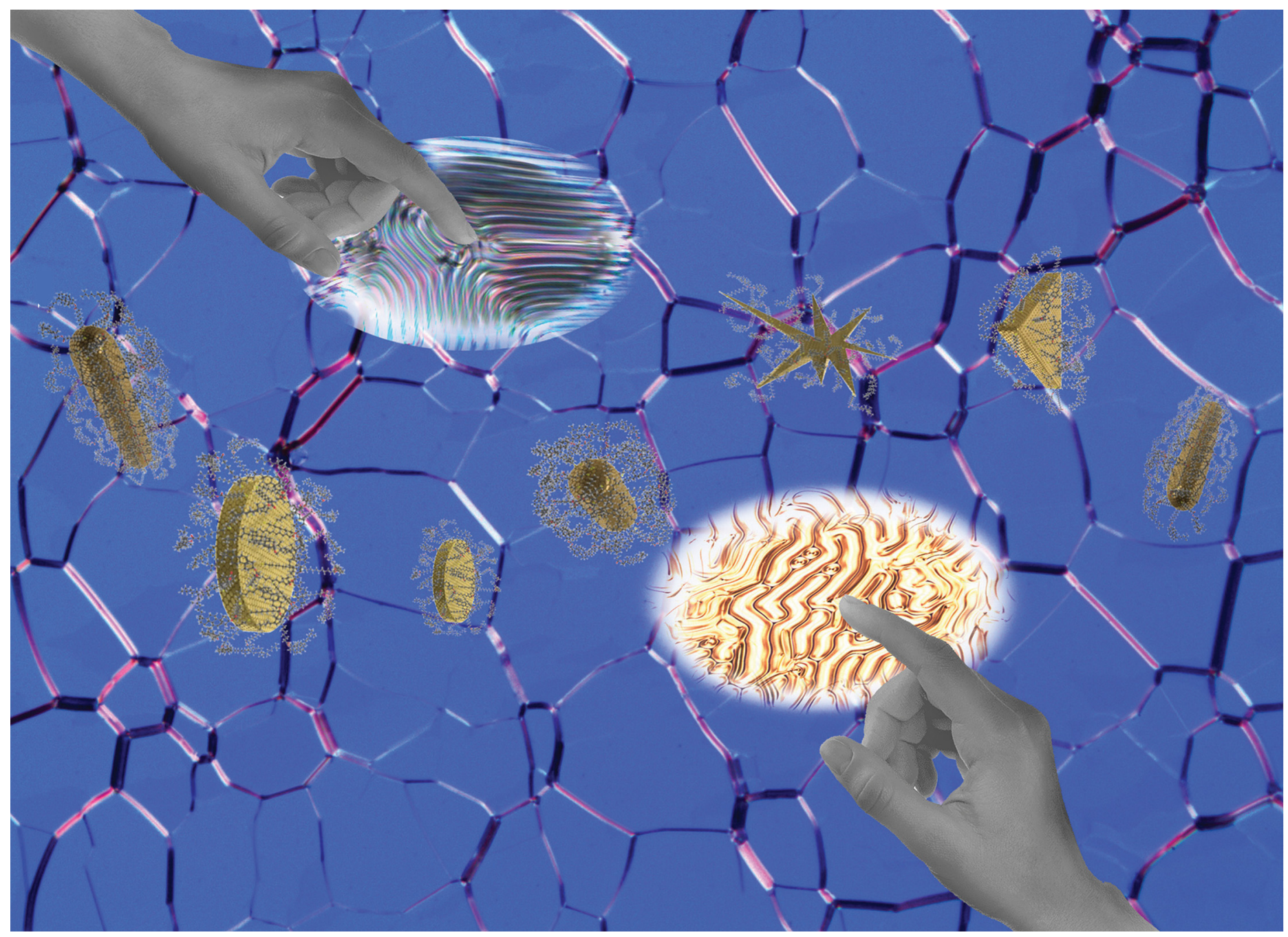

Showcasing research from the laboratories of Professors Torsten Hegmann and Claudio Zannoni, Advanced Materials and Liquid Crystal Institute, Kent State University, Kent. (Ohio), USA and Department of Industrial Chemistry "Toso Montanari", University of Bologna, Bologna, Italy.

The significance of nanoparticle shape in chirality transfer to a surrounding nematic liquid crystal reporter medium

As it turns out, the efficacy of chirality transfer may be understood by surprisingly simple geometric considerations. A combination of theory and experiment helped elucidate a mechanism based on the product of an established pseudoscalar chirality indicator and a scalar geometric shape compatibility factor. The experimental system is based on precisely engineered gold nanoshapes in a nematic liquid crystal solvent reporting medium. The predictive power of this approach may find use in macromolecular and biological systems, from chiral metamaterials to understanding nature's innate ability to transfer homochirality.
As featured in:

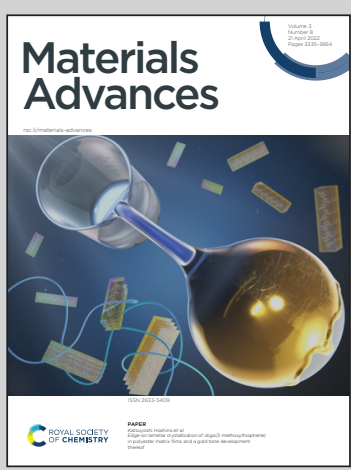

See Claudio Zannoni,

Torsten Hegmann et al.,

Mater. Adv., 2022, 3, 3346. 
Check for updates

Cite this: Mater. Adv., 2022, 3,3346

Received 27th January 2022, Accepted 3rd March 2022

DOI: $10.1039 / \mathrm{d} 2 \mathrm{ma} 00093 \mathrm{~h}$

rsc.li/materials-advances

\section{The significance of nanoparticle shape in chirality transfer to a surrounding nematic liquid crystal reporter medium}

\author{
Anshul Sharma, ${ }^{a b}$ Taizo Mori, (D) ${ }^{c}$ Ahlam Nemati, (D) ab Diana P. N. Gonçalves, (D) bd \\ Lara Querciagrossa, (D) Claudio Zannoni iD *e and Torsten Hegmann (D) *abdf
}

\begin{abstract}
Defined based on geometric concepts, the origin of biological homochirality including the single handedness of key building blocks, D-sugars and L-amino acids, is still heavily debated in many ongoing research endeavors. Origin aside, transmission and amplification of chirality across length scales are likely essential for the predominance of one handedness over the other in chiral systems and are attracting an unabated interest not only in biology but also in material science. To offer a measure for chirality and through-space chirality transfer, we here provide a report on recent progress toward the development of a suitable approach for an a priori prediction of chirality "strength" and efficacy of chirality transfer from a chiral solute to an achiral nematic solvent. We achieve this by combining an independently calculated, suitable pseudoscalar chirality indicator for the solute with another, independently calculated scalar solute-solvent shape compatibility factor. In our ongoing pursuit to put this approach to the test, we are advancing and refining a versatile experimental platform based on achiral gold nanoparticle cores varying in size, shape, and aspect ratio capped with monolayers of chiral molecules or on intrinsically chiral cellulose nanocrystals that serve as chiral solutes in an achiral nematic liquid crystal phase acting as a reporter medium. The pitch of the ensuing induced chiral nematic liquid crystal phase ultimately serves as a reporter medium that allows us to experimentally quantify and compare chirality and efficacy of chirality transfer.
\end{abstract}

\section{Introduction}

Chirality, defined as a geometric property of a group of points or atoms in space, or of any object that is not superimposable onto its mirror image, is ubiquitous at all scale levels ${ }^{1}-$ from subatomic particles ${ }^{2}$ to galaxies in space. ${ }^{3}$ For any system to be chiral, irrespective of any physical or chemical manifestation of said chirality, all that is necessary is the complete absence of improper rotations in the symmetry group of the system. Lord Kelvin defined chirality by the absence of mirror symmetry: "I

\footnotetext{
${ }^{a}$ Advanced Materials and Liquid Crystal Institute (AMLCI), Kent State University, Kent (OH) 44242-0001, USA. E-mail: thegmann@kent.edu

${ }^{b}$ Materials Science Graduate Program, Kent State University, Kent (OH) 44242-0001, USA

${ }^{c}$ Institute for Solid State Physics, The University of Tokyo, Tokyo, Japan

${ }^{d}$ Department of Chemistry and Biochemistry, Kent State University, Kent (OH) 44242-0001, USA

${ }^{e}$ Dipartimento di Chimica Industriale "Toso Montanari" and INSTM, Università di Bologna, Viale Risorgimento 4, IT-40136 Bologna, Italy. E-mail: claudio.zannoni@unibo.it

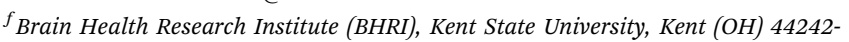
0001, USA
}

call any geometrical figure, or group of points, chiral, and say it has chirality, if its image in a plane mirror, ideally realized, cannot be brought to coincide with itself.," ${ }^{4}$ Considering its unquestionable significance in many areas of science ranging from cell-cell interactions, ${ }^{5}$ drug discovery, ${ }^{6}$ and catalysis ${ }^{7}$ to chiral optics ${ }^{8}$ and chiral nanomaterials, ${ }^{9-12}$ some key questions asked and addressed by many scientists are: Does chirality scale, can it be quantified even for abstract geometrical objects, and how does it vary solely as a function of shape? Kurt Mislow, a pioneer in this field of research, published one of the most detailed reports on purely geometric chirality metrics to quantify chirality. ${ }^{13}$ Now almost three decades ago, that report primarily focused on geometric figures in 2- and 3-D space representing molecules, rather than nanomaterials, but many of the concepts have since been applied to chiral nanostructures. Within the boundaries of each model or chirality measure, objects including molecules can be defined as "more" or "less" chiral. In due course, Mislow and colleagues concluded: "there remains the daunting challenge of bridging the gap between the results of chiral shape analysis and the world of experimental observables." ${ }^{\text {13 }}$

In this perspective, we summarize recent attempts by our teams to bridge this gap by showing that an appropriate 
pseudoscalar chirality index can fairly accurately predict the chirality transfer efficacy in an otherwise unrelated experimental system largely by assessing shape and shape congruency. While introducing the scope of and a classification for chirality measures, Mislow and colleagues noted that any "degree of chirality" scales with shape and should be invariant with the size of the object. ${ }^{13}$

Starting with this assumption, metal nanoparticles, particularly those with a gold core, continue to be fertile ground for testing shape-chirality correlations since gold nanoparticles can now be reproducibly synthesized in a very wide range of sizes and shapes with suitably narrow size and shape polydispersity. ${ }^{14}$ For the purpose of this discussion here, we will refer to these gold nanoparticles varying in size as well as shape, capped with a monolayer of chiral molecules as chiral nanoshapes.

\section{A chirality measure for nanoshapes}

\subsection{Basic concepts}

The intrinsic chirality of any given chiral shape (object or molecule) can be calculated using an absolute, as opposed to being relative to a reference, pseudoscalar chirality indicator ${ }^{15}$ - termed the average chirality index $\left\langle G_{\text {oa }}^{\mathrm{a}}\right\rangle \cdot \dagger^{16,17}$ For chiral nanoshapes, such as those of interest here, this index can be derived from coarse grained (CG) representations of the chiral ligand molecules suitably distributed on the nanoshape surface as we will see later. Experimental datasets correlated to the chirality of these nanoshapes are acquired by investigating dispersions of said chiral nanoshapes in an achiral nematic liquid crystal $(\mathrm{N}-\mathrm{LC})^{18}$ serving as a reporter medium. ${ }^{19}$ The induced chiral nematic LC ( $\mathrm{N}^{*}$-LC) phase allows a ranking of the efficacy of chirality transfer depending on the dimensions of the nanoshape via microscopically (visually)-observable and easily measurable helical pitch, $p$, values (Fig. 1). For any chiral nanoshape solute $X$ in an achiral N-LC phase $N, p$ of the induced $\mathrm{N}^{*}$-LC phase is inversely proportional to the concentration of the nanoshape, $c_{X}^{*}$, and to the helical twisting power $\beta^{X N}\left(\right.$ eqn (1)): ${ }^{20}$

$$
p \propto 1 /\left(\beta^{X N} c_{X}^{*}\right)
$$

Thus, $\beta^{X N}$ serves as a figure of merit or measure of chirality in these systems and is assumed to be proportional to the intrinsic chirality of the nanoshape (eqn (2)):

$$
\beta^{X N} \propto\left\langle G_{\text {oa }}^{\mathrm{a}}\right\rangle
$$

$\dagger$ This index depends only on geometric information, i.e., in this case the
position and orientation of the chiral ligands with respect to the nanoparticle
frame, and thus indirectly on the size, shape, and AR of the nanomaterial: ${ }^{16,17,24}$
$G_{o a}^{a}=\sum_{P} \begin{cases}\frac{\left[\left(\boldsymbol{r}_{i j} \times \boldsymbol{r}_{k l}\right) \cdot \boldsymbol{r}_{i l}\right]\left(\boldsymbol{r}_{i j} \cdot \boldsymbol{r}_{j k}\right)\left(\boldsymbol{r}_{j k} \cdot \boldsymbol{r}_{k l}\right)}{n\left(r_{i j} r_{j k} r_{k l}\right)^{2} r_{i l}} & \text { if } i<j<k<l \in[1, \ldots, n] \\ 0 & \text { otherwise }\end{cases}$ the total number of atoms (or beads), $\boldsymbol{r}_{i j}$ the vector between two atoms $i$ and $j$, with modulus corresponding to their distance $r_{i j}$, while $P$ indicates all permutations of $i, j, k, l$ atoms or beads.

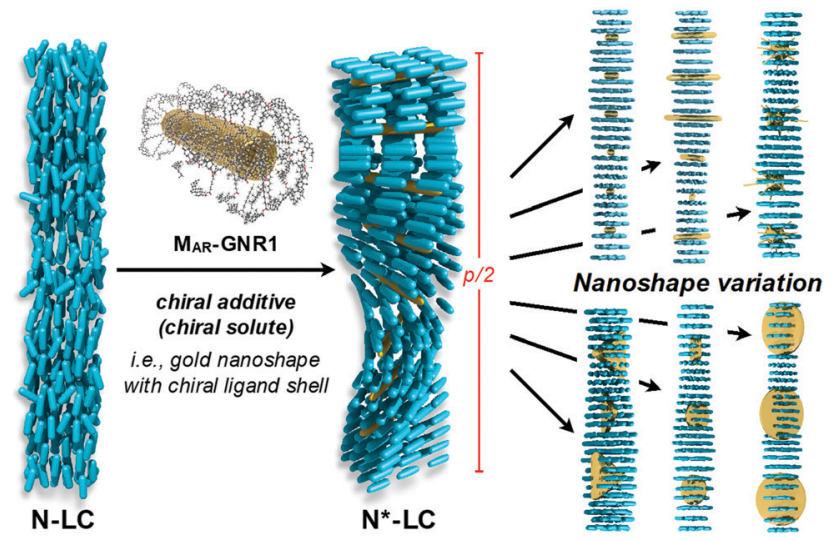

Fig. 1 Visual summary depicting the concept of using an induced chiral nematic liquid crystal ( $N^{*}-L C$ phase) as a reporter medium to study the chirality transfer efficacy (i.e., amplification across length scales) depending on the size, shape, and aspect ratio (AR) of gold nanocarriers capped with an identical chiral ligand shell; $p / 2$ refers to the helical half-pitch of the induced $N^{*}$-LC phase formed by doping the achiral N-LC phase. The right-side of the figure depicts different sizes and aspect ratios of the gold nanoshapes among $\mathrm{N}-\mathrm{LC}$ molecules in the induced $\mathrm{N}^{*}$-LC phase.

Early experiments by our teams focused on size effects of quasi-spherical (polyhedral) nanoparticles. Within each of the studied series of quasi-spherical gold nanoparticles (GNP), a particular GNP core diameter showed maximum values for both $\beta^{X N}$ and/or $\left\langle G_{\text {oa }}^{\mathrm{a}}\right\rangle{ }^{19}$ With the chirality emanating exclusively from a combination of spatial arrangement and chiral nature of the ligand shell molecules (considering an achiral metal core based on experimental i.e., spectroscopic evidence), the molar helical twisting power $\beta_{\text {mol }}$ defined in eqn (3):

$$
\beta_{\text {mol }}=1 /\left(p x_{\text {Ligand }} r\right)
$$

in which $p$ is the measured helical pitch, $x_{\text {Ligand }}$ the mole fraction of the chiral ligand in the N-LC mixture, and $r$ the enantiomeric purity of the chiral species), was used as the most accurate measure for the efficacy of chirality transfer to the $\mathrm{N}-\mathrm{LC}$ reporter medium.

\subsection{Early experimental nanoparticle systems}

For the first set of GNPs with core diameters of about 2, 5 and $10 \mathrm{~nm}$, capped with cholesterol-thiol or siloxane-networked cholesterol-thiol derivatives (Fig. 2), $\left|\beta_{\text {mol }}\right|$ was the largest (i.e., $p$ was the tightest) for $\mathrm{GNP}_{5}$ characterized by an average core diameter of $5.5 \mathrm{~nm}\left(\left|\beta_{\mathrm{mol}}\right|=178 \mu \mathrm{m}^{-1}\right.$ (we here report values for the absolute molar helical twisting power, $\left|\beta_{\text {mol }}\right|$, since the sign of $\beta_{\mathrm{mol}}$ indicates the handedness and not the magnitude; "+" for a right-handed and "_" for a left-handed helical distortion of the induced $\mathrm{N}^{*}$-LC phase). ${ }^{19}$

In contrast, $\mathrm{GNP}_{10}$ with the largest core diameter did not induce an apparent $\mathrm{N}^{*}$-LC phase at any concentration judging from textural analysis data obtained by cross-polarized optical microscopy (cPOM) with the LC samples confined between substrates with varying surface boundary conditions (including homeotropic anchoring or a top surface formed by air). The observed Schlieren textures were indistinguishable from 


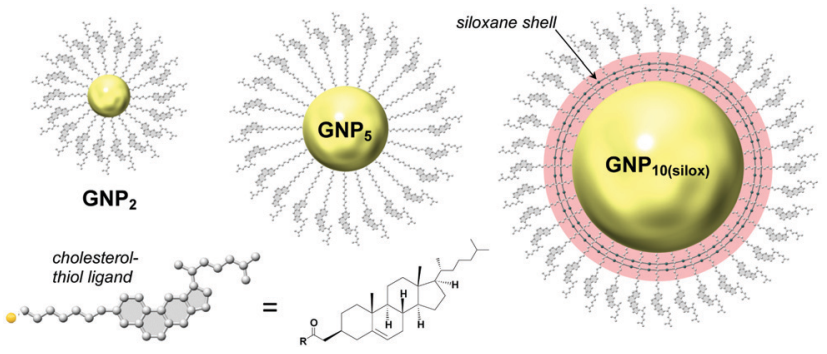

Fig. 2 Studies focusing on the effects of core diameter and presence (absence) of a chiral bias during GNP formation. $\mathrm{GNP}_{2}$ and $\mathrm{GNP}_{5}$ (the subscript number indicating the average core diameter) are capped with cholesterol-thiol ligands; $\mathrm{GNP}_{10(\text { silox) }}$ is capped with a siloxane shell using 3-mercaptopropyltrimethoxysilane (MPS) that is further endfunctionalized with cholesterol. Reproduced from and adapted with permission from ref. 19 - Copyright (2014), American Chemical Society.

textures commonly seen for achiral N-LC phases, thus indicating $p \rightarrow \infty$ and, as a consequence, $\beta_{\text {mol }} \rightarrow 0 . \mathrm{GNP}_{2}$ showed a value of $\left|\beta_{\text {mol }}\right|=16 \mu \mathrm{m}^{-1}$; about one order of magnitude lower than the value detected for $\mathrm{GNP}_{5}$ but compared reasonably well to some commercially available organic chiral additives developed to induce $\mathrm{N}^{*}$-LC phases with $\left|\beta_{\text {mol }}\right|$ values ranging from about 10 to $40 \mu \mathrm{m}^{-1}$. ${ }^{19}$

Another GNP series investigated the role of axially chiral ligands. GNPs capped with three sets of $(R)$ - and $(S)$-enantiomers based on axially chiral $1,1^{\prime}$-binaphthyl-thiol derivatives that differed only in the length of a non-tethered aliphatic chain were investigated as chiral solutes in the same N-LC host (Fig. 3). ${ }^{21}$ The variation in length of the non-tethered aliphatic chain in the 2 '-position, potentially due to reasons of varying dihedral angles in solution and the resulting changes in steric demand (or due to differences in solubility) gave rise to some variation in GNP core diameter during synthesis (overall ranging from about 1.1-2.5 nm in core diameter). The binaphthyl-thiol-capped GNP with the

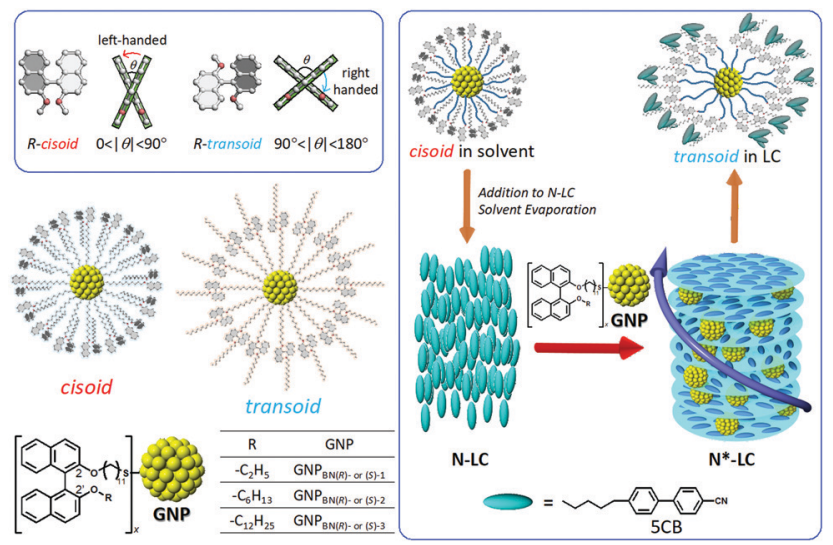

Fig. 3 Series of GNPs capped with 1,1'-binaphthyl-thiol ligands differing in the length of the non-tethered aliphatic chain in the $2^{\prime}$-position $((R)$ - and $(S)$-enantiomers) tested as chiral solutes in the $\mathrm{N}$-LC phase formed by 5 CB (4'-pentyl-4-biphenylcarbonitrile). While cisoid-conformations were observed in organic solvents, transoid-conformations were confirmed in the induced $\mathrm{N}^{*}$-LC phase. Reproduced from and adapted with permission from ref. 21 - Copyright (2016), American Chemical Society. largest core diameter of about $2.5 \mathrm{~nm}\left(\mathrm{GNP}_{\mathrm{BN}(R)-1}\right)$ turned out to be just slightly the strongest chiral inducer of this series with $\left|\beta_{\text {mol }}\right|=66 \mu \mathrm{m}^{-1}$. However, in light of comparatively smaller differences in core diameter vis-à-vis the earlier series ${ }^{19}$ overall, all six GNPBN essentially showed identical $\left|\beta_{\text {mol }}\right|$ values, ranging from $\left|\beta_{\mathrm{mol}}\right|=61 \mu \mathrm{m}^{-1}$ to $\left|\beta_{\mathrm{mol}}\right|=66 \mu \mathrm{m}^{-1}$. Most noteworthy, all GNPs discussed thus far (with the exception of $\mathrm{GNP}_{10}$ ) well outperformed the free cholesterol-thiol and $1,1^{\prime}$-binaphthyl-thiol ligands with respect to their ability to helically distort the initially achiral N-LC, i.e., induce a significantly lower pitch (higher $\left.\left|\beta_{\text {mol }}\right|\right)$ at any identical concentration of the chiral molecules in the N-LC matrix. ${ }^{19,21}$

\subsection{Desymmetrization from GNPs to GNRs (gold nanorods)}

Our initial hypothesis was that the observed enhancement of this through-space chirality transfer was caused by long-range, through-space interactions between the chiral molecules and the plasmonic nanostructures exemplified by spectroscopically observed enhanced anisotropy or Kuhn's dissymmetry factors $g$ (eqn (4)): ${ }^{22}$

$$
g=\Delta \varepsilon / \varepsilon
$$

where $\Delta \varepsilon$ and $\varepsilon$ are the molar circular dichroism and molar extinction coefficient, respectively) here for chiral molecules in the vicinity of plasmonic nanostructures. Even higher $g$ values were reported when such quasi-spherical GNPs were replaced by elongated GNRs, ${ }^{23}$ which, based on our hypothesis, should lead to increased $\left|\beta_{\text {mol }}\right|$ values when anisometric GNRs capped with the same chiral molecules are dispersed as a chiral solute in the identical N-LC host.

Experimental data indeed confirmed our hypothesis,${ }^{24}$ and each of the two GNRs differing in their aspect ratio (AR) and capped with a siloxane networked cholesterol-thiol ligand shell led to significantly higher $\left|\beta_{\text {mol }}\right|$ values (Fig. 4). $\mathrm{M}_{\mathrm{AR}}-\mathrm{GNR} 1\left(\mathrm{M}_{\mathrm{AR}}\right.$ here standing for the medium aspect ratio of $\mathrm{AR}=4.3$ with the transversal cross-sectional diameter of this GNR of $d=10 \mathrm{~nm}$ closely matching the diameter of $\mathrm{GNP}_{10}$ ) produced a value of $\left|\beta_{\text {mol }}\right|=1064 \mu \mathrm{m}^{-1}$. Similarly, $\mathrm{M}_{\mathrm{AR}}-\mathrm{GNR} 2$ with $\mathrm{AR}=2.2$ induced an $\mathrm{N}^{*}$-LC phase with a value of $\left|\beta_{\mathrm{mol}}\right|=895 \mu \mathrm{m}^{-1}$. Remarkably, the trends of independently calculated or acquired $\left\langle G_{\text {oa }}^{\mathrm{a}}\right\rangle$ and $\left|\beta_{\text {mol }}\right|$ values, respectively, for this $\mathrm{GNP}_{2-10} / \mathrm{M}_{\mathrm{AR}}$-GNR series matched almost perfectly (Fig. 5). Our, for the most part, naïve conclusions from these first couple of datasets were that desymmetrizing the shape of the nanocarrier and increasing its AR will increase $\left\langle G_{\text {oa }}^{\mathrm{a}}\right\rangle$ and $\left|\beta_{\text {mol }}\right|{ }^{24}$

This was further supported by the considerable amplification of the chirality transfer by $\mathrm{M}_{\mathrm{AR}^{-}-\mathrm{GNR} 1 \text { (the more effective chiral }}$ inducer of the GNRs tested thus far) capped with the identical series of axially chiral binaphthyl-thiol ligands described earlier.

For this particular $\mathrm{M}_{\mathrm{AR}}-\mathrm{GNR} 1_{\mathrm{BN}}$ series, ${ }^{25}$ the $\left|\beta_{\mathrm{mol}}\right|$ values, now strongly depending on the length of the non-tethered aliphatic chain in the $2^{\prime}$-position on the $1,1^{\prime}$-binaphthyl unit, ranged from $\left|\beta_{\text {mol }}\right|=192 \mu \mathrm{m}^{-1}$ to a record $\left|\beta_{\mathrm{mol}}\right|=1312 \mu \mathrm{m}^{-1}$. Here again, independent calculations of $\left\langle G_{\text {oa }}^{\mathrm{a}}\right\rangle$ predicted these experimental trends both with respect to magnitude of $\left|\beta_{\mathrm{mol}}\right|$ as well as the sign (aka handedness) of the induced $\mathrm{N}^{*}$-LC phase (Fig. 6). ${ }^{25}$ 
a
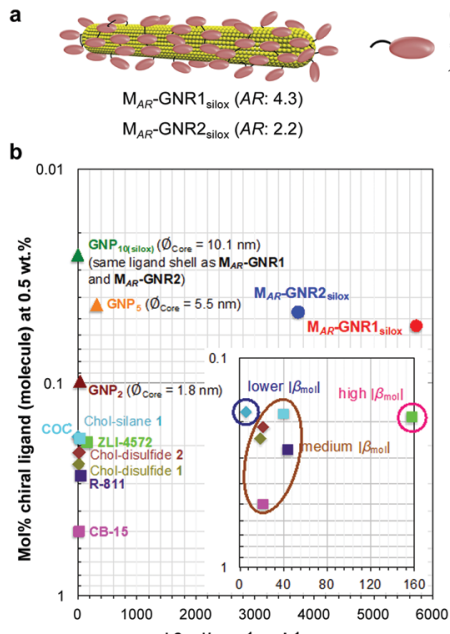

$\left|\boldsymbol{\beta}_{\mathrm{mol}}\right| / \mathrm{\mu m}^{-1} \mathrm{~mol}^{-1}$

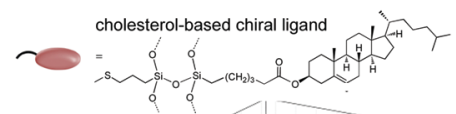

c

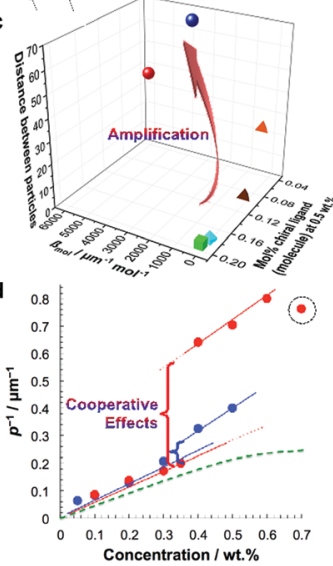

Fig. 4 Amplification of the chirality transfer efficacy by desymmetrization. (a) GNRs with a cholesterol end-functionalized siloxane ligand shells show significantly elevated values of $\left|\beta_{\text {mol }}\right|$ in comparison to GNPs as shown in plots (b) and (c). ZLI-4572, COC, R-811, and CB15 are commercially available organic chiral dopants; the Chol-silane and Chol-disulfides are the neat organic ligand molecules tested as chiral additives, $\varnothing$ core is the core diameter of the GNP. $\left|\beta_{\text {mol }}\right|$ values here used the molar concentration of the entire ligand-capped GNR and thus the SI unit $\left[\mu \mathrm{m}^{-1} \mathrm{~mol}^{-1}\right]\left(\left|\beta_{\text {mol }}\right|\right.$ values described in the text as well as in Table 1 use the more correct and dimensionless mole fraction of the chiral ligand shell molecules only since the GNR core is achiral; eqn (3)). (d) Cooperative effects among the admixed GNRs are supported by analyzing plots of the inverse pitch (1/p) $v s$. the $M_{A R}-G N R$ concentration; the slope increases at a threshold concentration rather than plateauing as commonly observed for chiral additives in induced $\mathrm{N}^{*}$-LC phases (green dotted curve) until a concentration when the GNRs begin the aggregate (dotted circle at $C_{M_{A R}-G N R 1_{\text {silox }}}=0.7 \mathrm{wt} \%$ ). Reproduced from and adapted with permission from ref. 24 - Copyright (2018), Springer-Nature.

For all the nanoshapes described so far, the working principle for any amplification of the efficacy of chirality transfer always appeared to be that the chiral ligand shell molecules act as a joined force or network, thereby amplifying chirality transfer throughout the N-LC medium both locally (to N-LC molecules in direct vicinity of the suspended GNRs) and throughout the NLC bulk. Freeze-fracture transmission electron microscopy (TEM) experiments, showing arrays of appropriately spaced (based on simple volume fraction calculations) and seemingly twisted with respect to each other $\mathrm{M}_{\mathrm{AR}}$-GNR2, lend support for an additional contribution to this efficacious chirality transfer (Fig. 7). ${ }^{24}$ It appeared reasonable to assume that cooperative effects among the now twisted GNRs would further amplify chirality transfer, and such cooperative effects were experimentally seen in the plots of $1 / p v s$. the concentration of the GNRs $\left(c_{X}^{*}\right)$ in the N-LC. At a distinct threshold value of $c_{X}^{*}$, the slope of the curve would abruptly rise with increasing $c_{X}^{*}$ rather than plateau as normally observed for chiral additives in N-LCs. ${ }^{24}$ One may think of this contribution as a feedback loop at and above the threshold concentration: a spatially massive (when compared to the N-LC building blocks, i.e., N-LC molecules) and strong chiral inducer amplifies its own twist and thus shortens $p$ in the surrounding matrix, which, in turn leads to an

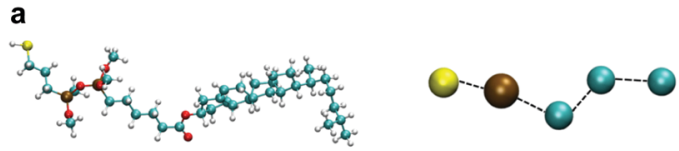

b
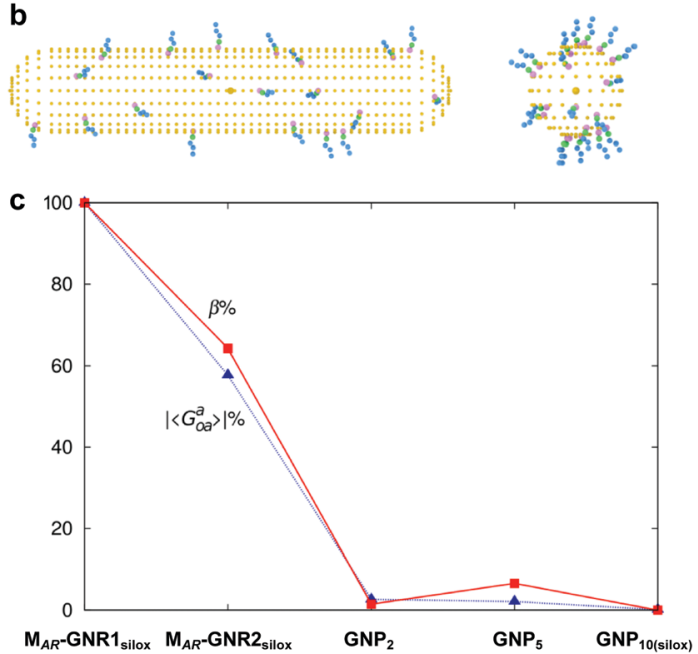

Fig. 5 (a) Atomistic and coarse-grained (CG) representation of the ligand molecule, (b) $M_{A R}-G N R 1$ spherocylindrical and $G_{N P} P_{10}$ models each decorated with 20 CG ligands randomly distributed. (c) Trend of averaged chirality indicator $\left|G_{\circ a}^{a}\right|$ in comparison to the trend experimentally determined for $\left|\beta_{\text {moll }}\right|$ for $G_{N P s_{2-10}}$ as well as $M_{A R}-G N R 1$ and $M_{A R}-G N R 2$. Values of chirality have been obtained for 20000 random configurations on the GNPs and 1800 selected configurations on the GNRs. Reproduced from and adapted with permission from ref. 24 - Copyright (2018), Springer-Nature.

increased torque to the GNRs that shortens $p$ and so on and so forth.

\subsection{Variations in AR, other shapes, and shape matching}

Equipped with these pieces of the puzzle, the next question in our quest was: How do shape and AR of the nanocarrier of chiral information impact chirality transfer? Moreover, reflecting on our appreciation that an independently calculated $\left\langle G_{\text {oa }}^{\mathrm{a}}\right\rangle$ can predict experimental trends of $\mid \beta_{\text {mol }}$, we put this system to the test with a significant expansion of the scope of our research by focusing on more pronounced shape and AR variations. ${ }^{26}$

This was ultimately accomplished by comparing calculations of a shape-corrected $\left\langle G_{\text {oa }}^{\text {a }}\right\rangle$, described next, with the experimental values gathered for $\left|\beta_{\text {mol }}\right|$ for a larger set of gold nanoshapes now including nanoprisms (GNPR), nanodisks (GND), nanostars (GNS) as well as GNRs differing in AR. All gold nanoshapes were monolayer-capped with the identical cholesterol-thiol ligand shell shown in Fig. 2. The dimensions as well as $\left|\beta_{\text {mol }}\right|$ values for these and all other nanoshapes discussed in this perspective are summarized in Table 1.

To account for a shape compatibility dependence of the chirality transfer, our new model assumed that there is a combination of the chiral solute's intrinsic chirality and its ability to twist the N-LC that contributes to the transfer chirality across length scales. For a chiral solute (nanoshape) X, we assumed that $\beta^{X N}$ can be written as:

$$
\beta^{X N} \propto G^{X} S^{X N}
$$




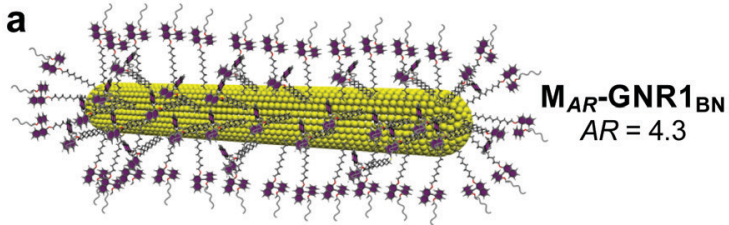

b

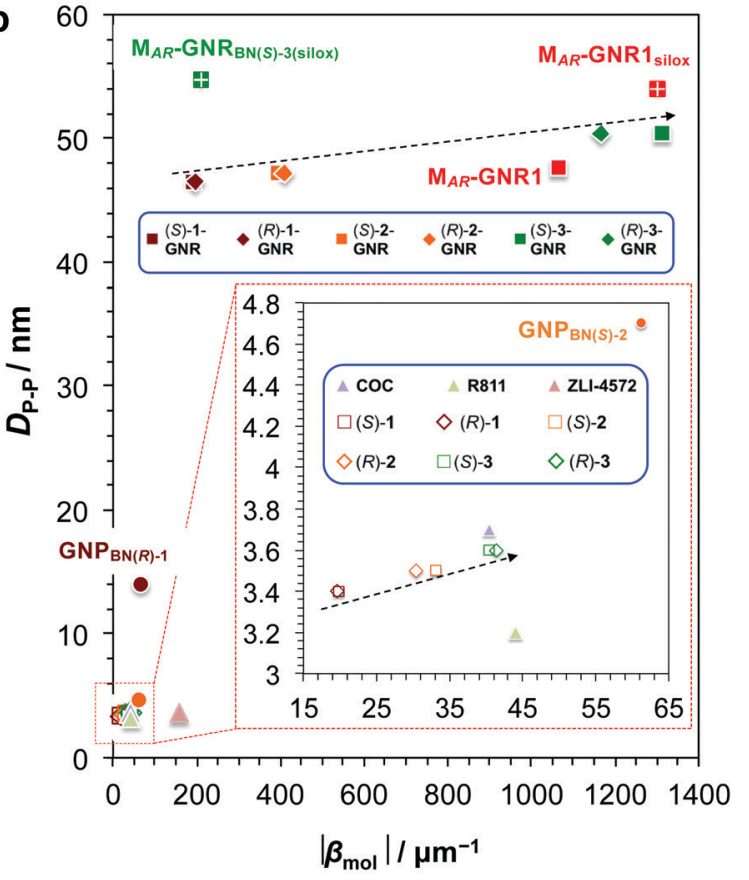

C

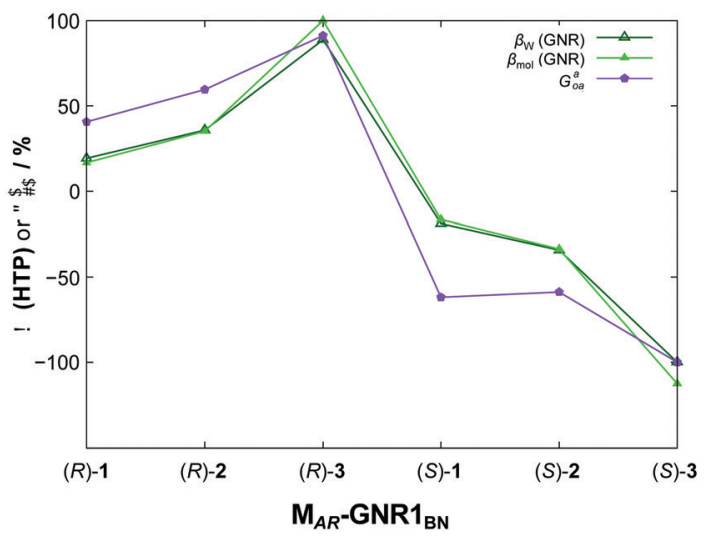

Fig. 6 (a) Schematic depiction of the $M_{A R}-G N R 1_{B N}$ series with the 2,2'substituted 1,1'-binaphthyl-thiol ligands in the transoid conformation formed in the induced $N^{*}$-LC phase. (b) Chirality efficiency plot: calculated particle-particle distances, $D_{\mathrm{P}-\mathrm{P}}$, vs. $\left|\beta_{\text {mol }}\right|$. The most efficient chiral GNR solutes occupy the top right corner of this plot, the bottom lower corner the least efficient ones. Inset (red dashed box) shows the section highlighted in the lower left part of the plot (data points are either directly identified with the abbreviation of the nanoshape or highlighted in blue boxes. (c) Values of the chirality index, $\left\langle G_{o a}^{a}\right\rangle$, for 20 binaphthyl thiol ligand molecules bound to the $M_{A R}-G N R 1$ compared to the values of the experimentally determined HTP data, $\left|\beta_{\mathrm{w}}\right|$ (helical twisting power based on the weight fraction of the chiral BN ligands in the $\mathrm{N}-\mathrm{LC}$ ), and $\left|\beta_{\text {mol }}\right|$ (with the largest values were set to $100 \%$ ). Reproduced from and adapted with permission from ref. 25 - Copyright (2019), American Chemical Society.

in which $G^{X}$ is the chirality indicator $\left\langle G_{\text {oa }}^{\text {a }}\right\rangle$ of the nanoshape and $S^{X N}$ a compatibility factor representing a chiral solute - achiral

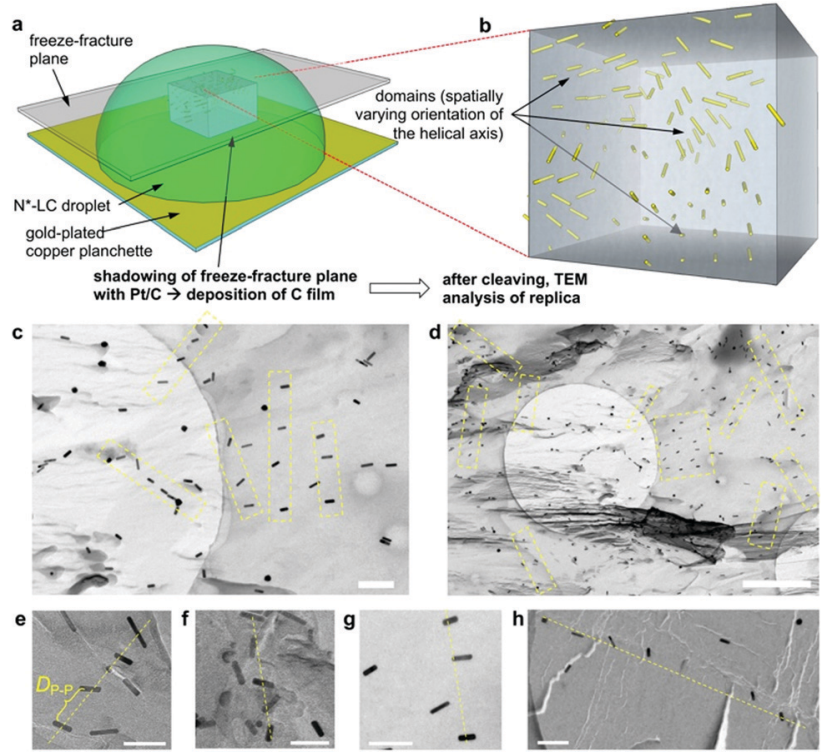

Fig. 7 (a) Schematic depiction of the freeze-fracture (FF) method to prepare the TEM specimen. (b) A representative voxel of the $N^{*}-L C$ droplet showing the multi-domain structure via the embedded GNRs. (c) and (d) FF-TEM images of the induced $N^{*}$-LC phase of Felix-2900-03 containing $0.5 \mathrm{wt} \% \mathrm{M}_{\mathrm{AR}}-\mathrm{GNR} 2_{\text {silox }}$ (scale bars: (c) $200 \mathrm{~nm}$ and (d) $1 \mu \mathrm{m}$, apparent circles are from the TEM grids). To obtain these images, it was important that the replica captured most of the $M_{A R}-G N R s$ on the fractured surface, thereby providing a direct visualization of the GNRs in the bulk material. Areas highlighted by a yellow box and (e-h) select areas from many of the obtained FF-TEM images show GNR arrays (often twisted) with an average separation closely matching the theoretically calculated particle-particle distances, $D_{\mathrm{P}-\mathrm{p} \text {, }}$ of $c a$. $90-100 \mathrm{~nm}$ by assuming well-dispersed GNRs in the induced $N^{*}$ - LC matrix (scale bars in $(e-h)$ : $100 \mathrm{~nm}$ ). Reproduced from and adapted with permission from ref. 24 - Copyright (2018), SpringerNature.

solvent transmission coefficient of the chiral nanoshape solute $\mathrm{X}$ to the achiral nematic mesogen $\mathrm{N}$. Here, we employed for the first term the maximum chirality indicator (Fig. 8a); for $S^{X N}$ we now introduced a shape-complementarity model to quantify the shape resemblance (or shape incongruity) between the N-LC molecule serving as host and the chiral ligand surface-modified nanoshape. ${ }^{26}$

The use of $\left|G_{\mathrm{oa} \text { max }}^{\mathrm{a}}\right|$ was justified by the fact that the HTP data were calculated from experimental pitch measurements, which are particularly sensitive to the highest rather than average chirality values (particularly for a system containing various contributions of different chirality).

Hereby we further assumed that $S^{X N}$ is related to the inverse difference between the 2-D isoperimetric ratios $\left(\mathrm{IPR}_{2 \mathrm{D}}\right.$; defined as $\mathrm{IPR}_{2 \mathrm{D}}=\ell^{2} / A$, with $\ell$ being the length of the perimeter and $A$ the $2 \mathrm{D}$ shape surface area) of solute and solvent, $1 / \mathrm{IPR}_{2 \mathrm{D}}{ }^{X N}=$ $1 /\left(\mathrm{IPR}_{2 \mathrm{D}}{ }^{X}-\mathrm{IPR}_{2 \mathrm{D}}{ }^{N}\right)$. The degree of correlation between $S^{X N}$ and $\beta^{X N}$ (i.e., $\left.\left|\beta_{\mathrm{mol}}\right|\right)$ will then provide direct insights into the importance of shape complementarity in chirality transfer. $S^{X N}$, of course, is a scalar quantity such that the orientation of one nanoshape with respect to another is non-zero and scales invariantly under spatial transformation, even in the achiral case. We defined $S^{X N}$ as the absolute value of the inverse 
Table 1 Comparison of experimentally determined molar helical twisting power, $\mid \beta_{\text {mol }}$, values of the chiral ligand-capped gold nanoshapes as solutes in the N-LC 5CB. Data from ref. 19, 21 and 24-26

\begin{tabular}{|c|c|c|c|}
\hline \multirow{2}{*}{$\frac{\text { Nanoshape }^{a}}{\mathrm{GNP}_{2}}$} & \multicolumn{2}{|c|}{$\begin{array}{l}\text { Nanoshape Core Dimensions }{ }^{b} \\
(\mathrm{~nm})(d=\text { diameter, } w=\text { width, } \\
h=\text { height, } l=\text { length, } \\
\mathrm{AR}=\text { aspect ratio })\end{array}$} & \multirow{2}{*}{$\frac{\mid \begin{array}{l}\left|\beta_{\mathrm{mol}}\right| \\
\left(\mu \mathrm{m}^{-1}\right)\end{array}}{16}$} \\
\hline & $d=1.8$ & $\mathrm{AR} \sim 1^{c}$ & \\
\hline $\mathrm{GNP}_{5}$ & $d=5.5$ & & 178 \\
\hline $\mathrm{GNP}_{10}$ & $d=10.0$ & & 0 \\
\hline $\mathrm{GNP}_{10 \text { (silox) }}$ & $d=10.0$ & & 0 \\
\hline $\mathrm{GNP}_{\mathrm{BN}(R)-1}$ & $d=2.5$ & & $61-66$ \\
\hline $\mathrm{GNP}_{\mathrm{BN}(S)-1}$ & $d=1.7$ & & \\
\hline $\mathrm{GNP}_{\mathrm{BN}(R)-2}$ & $d=2.1$ & & \\
\hline $\mathrm{GNP}_{\mathrm{BN}(S)-2}$ & $d=1.1$ & & \\
\hline $\mathrm{GNP}_{\mathrm{BN}(R)-3}$ & $d=1.2$ & & \\
\hline $\mathrm{GNP}_{\mathrm{BN}(S)-3}$ & $d=1.1$ & & \\
\hline $\mathrm{M}_{\mathrm{AR}}-\mathrm{GNR} 1$ & $d=10.0 ; l=43.0$ & $\mathrm{AR}=4.3$ & 1064 \\
\hline $\mathrm{M}_{\mathrm{AR}}-\mathrm{GNR} 1_{\text {silox }}$ & & & 1303 \\
\hline $\mathrm{M}_{\mathrm{AR}}-\mathrm{GNR} 1_{\mathrm{BN}(R)-1}$ & & & 196 \\
\hline $\mathrm{M}_{\mathrm{AR}}-\mathrm{GNR} 1_{\mathrm{BN}(S)-1}$ & & & 192 \\
\hline $\mathrm{M}_{\mathrm{AR}}-\mathrm{GNR} 1_{\mathrm{BN}(R)-2}$ & & & 410 \\
\hline $\mathrm{M}_{\mathrm{AR}}-\mathrm{GNR} 1_{\mathrm{BN}(S)-2}$ & & & 393 \\
\hline $\mathrm{M}_{\mathrm{AR}}-\mathrm{GNR} 1_{\mathrm{BN}(R)-3}$ & & & 1166 \\
\hline $\mathrm{M}_{\mathrm{AR}}-\mathrm{GNR} 1_{\mathrm{BN}(S)-3}$ & & & 1312 \\
\hline $\mathrm{M}_{\mathrm{AR}}-\mathrm{GNR} 1_{\mathrm{BN}(S)-3 \text { silox }}$ & & & 210 \\
\hline $\mathrm{M}_{\mathrm{AR}}-\mathrm{GNR} 2_{\text {silox }}$ & $d=23.5 ; l=51.0$ & $\mathrm{AR}=2.2$ & 896 \\
\hline $\mathrm{L}_{\mathrm{AR}}-\mathrm{GNR}$ & $d=15.2 ; l=25.0$ & $\mathrm{AR}=1.7$ & 53 \\
\hline $\mathrm{H}_{\mathrm{AR}}-\mathrm{GNR}$ & $d=12.5 ; l=87.0$ & $\mathrm{AR}=8.5$ & 18 \\
\hline $\mathrm{L}_{\mathrm{AR}}-\mathrm{GND}$ & $d=79.0 ; h=21.6$ & $\mathrm{AR}=3.7$ & 37 \\
\hline $\mathrm{H}_{\mathrm{AR}}-\mathrm{GND}$ & $d=45.0 ; h=7.4$ & $\mathrm{AR}=6.1$ & 43 \\
\hline GNS & $d_{\text {core }}=60.0 ; l_{\text {spikes }}=69.0$ & $-{ }^{d}$ & 105 \\
\hline GNPR & $l_{\text {edoe }}=50.0 ; h=12.5$ & $\mathrm{AR}=5.3$ & 216 \\
\hline 5CB (N-LC) & $l=1.8 ; w=0.45$ & $\mathrm{AR}=4.0$ & - \\
\hline
\end{tabular}

${ }^{a}$ All nanoshapes are capped with a thiolate ligand shell unless otherwise noted, e.g., the subscript "silox" refers to nanoshapes capped with a siloxane conjugated 3-mercaptopropylsilyloxy-ligand shell endfunctionalized with aliphatic cholesteryl ester groups via a siloxane condensation reaction; $\mathrm{BN}=1,1^{\prime}$-binaphthyl; all other nanoshapes are capped with a cholesterol-thiol; $\mathrm{L}_{\mathrm{AR}}, \mathrm{M}_{\mathrm{AR}}$ and $\mathrm{L}_{\mathrm{AR}}$ refer to large, medium or low aspect ratio, respectively. ${ }^{b}$ Calculated AR values here are not considering the thickness of the ligand monolayer. Those referred to in the text used for the calculation of the 2-D isoperimetric ratios, $I_{P R_{2 D}}$, consider the ligand shell of the chiral ligand-capped nanoshapes and thus differ from those listed here for the nanoshape cores. ${ }^{c}$ Assuming quasi-spherical overall shapes for polyhedral GNPs. ${ }^{d}$ The AR cannot be easily calculated for GNS; thus far the only concave shape investigated.

difference between the $\mathrm{IPR}_{2 \mathrm{D}}$ of each nanoshape and the $\mathrm{IPR}_{2 \mathrm{D}}$ of the N-LC host molecule 5CB (eqn (6)):

$$
S^{X N} \sim\left|\frac{1}{\Delta\left(\mathrm{IPR}_{2 \mathrm{D}}\right)}\right|
$$

i.e., the closest match between their respective 2-D shapes was set to $100 \%$. The $2-\mathrm{D}$ approach proved to be significantly more sensitive than the corresponding 3-D IPR approach considering a comparison of the trends of $I_{P R}$ and $I P R_{3 D}$ (defined as $\mathrm{IPR}_{3 \mathrm{D}}=A^{3} / V^{2}$, where $V$ is the volume of the nanoshape) $v s$. $\left|\beta_{\text {mol }}\right|$ shown in Fig. $8 \mathrm{~b}$ and c. $\mathrm{IPR}_{2 \mathrm{D}}$ clearly shows a very close match to the experimentally derived $\left|\beta_{\text {mol }}\right|$ values (Fig. $8 \mathrm{~b}$ ). Hence, the chirality transfer is experienced by the $5 \mathrm{CB}$ host molecules from the 2-D projection of the suspended nanoshapes, i.e., from the distribution of chiral molecules on the nanoshape surface they are in direct contact with. Plotting
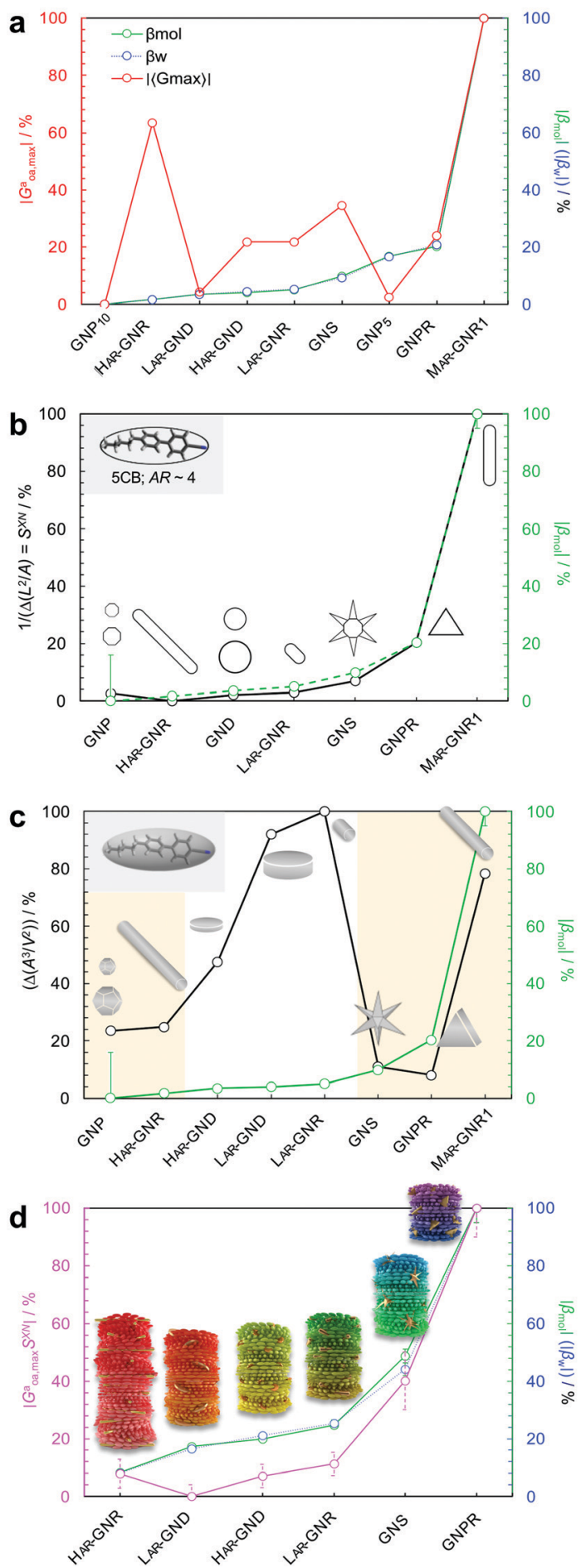

Fig. 8 Plots showing the trends of: (a) the maximum chirality indicator $\left|G_{\mathrm{o} a \text {,max }}^{\mathrm{a}}\right|$ in comparison to the experimentally derived values for $\left|\beta_{\mathrm{w}}\right|$ and $\mid \beta_{\text {mol }}$ (with the largest value set to $100 \%$ and the lowest to $0 \%$-here $\mathrm{GNP}_{10}$ with $\beta_{\mathrm{mol}}=\beta_{\mathrm{w}}=0$, and every other value-and every other value weighted accordingly) for the calculated values of $\left|\beta_{\text {mol }}\right|$ see Table 1 , (b) $1 /\left(\operatorname{IPR}_{2 \mathrm{D}}\right)$ and (c) $1 /\left(I_{3 P}\right.$ ) each in comparison to $\mid \beta_{\text {mol }}$ (yellow shaded areas in (c) signify some correlation in trends); values for IPR $2 \mathrm{D}$ and IPR $3 \mathrm{D}$ use the adjusted $A R$ values considering the thickness of the ligand shell. (d) Plot showing the trend of the shape compatibility-corrected maximum chirality indicator $\left|G_{\mathrm{oa}, \max }^{\mathrm{a}}{ }^{X N}\right|$ for a subset of nanoshapes in comparison to $\left|\beta_{\mathrm{w}}\right|$ and $\left|\beta_{\text {mol }}\right| \cdot L_{A R}, M_{A R}$, and $H_{A R}$ here refer to low, medium and high aspect ratio, respectively. Reproduced from and adapted with permission from ref. 26 - Copyright (2022), AAAS. 


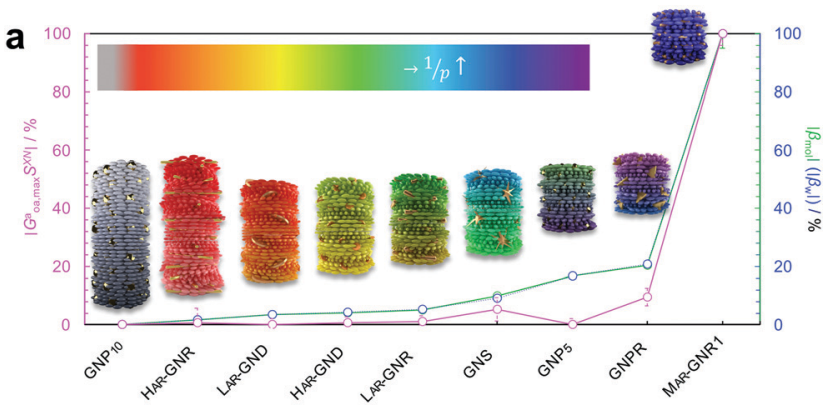

b
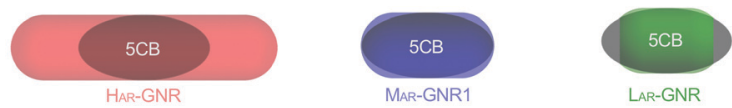

Fig. 9 (a) Plot showing trends of the shape compatibility-corrected maximum chirality indicator $\left|G_{\mathrm{oa}, \max }^{\mathrm{a}} S^{X N}\right|$ for the full set of nanoshapes, now including the powerful chiral inducer $M_{A R}-G N R 1$, in comparison to the experimentally derived values for $\left|\beta_{\mathrm{w}}\right|$ and $\left|\beta_{\text {mol }}\right|$. Models of the induced $N^{*}$-LC phase show a complete $360^{\circ}$ rotation of the local director leading to a tighter pitch from left to right depending on the size, shape, and AR of the suspended nanoshape (for $\mathrm{GNP}_{10}: \mathrm{p} \rightarrow \infty$ ). (b) Visualization showing the varying degree of shape compatibility among GNRs featuring different $A R$ values with the 2-D projection of $5 C B$ scaled in size to match each GNR width. Reproduced from and adapted with permission from ref. 26 Copyright (2022), AAAS

the shape compatibility-corrected $\left|G_{\text {oa, } \max }^{\mathrm{a}} S^{X N}\right|$ against the experimentally obtained values shows extraordinary close correlation across almost all nanoshapes with respect to $\left|\beta_{\mathrm{w}}\right|$ and $\left|\beta_{\text {mol }}\right|$ (Fig. 8d and 9a), most noteworthy all in the absence of any fitting parameter.

The importance of the shape coupling or shape compatibility factor $S^{X N}$ is most apparent when the AR of $X$ is changing within a series of equal-shaped nanoshape solutes in the same $N$. This concept is best visualized by sketches shown in Fig. 9b, where the shape compatibility among GNRs, $\mathrm{H}_{\mathrm{AR}^{-}} \mathrm{GNR}, \mathrm{M}_{\mathrm{AR}^{-}}$ GNR1 and $\mathrm{L}_{\mathrm{AR}}-\mathrm{GNR}$ proves the utility of introducing $S^{X N}$.

\section{Summary and outlook}

Collectively, all our experimental data support the notion that size, shape, and AR of chiral ligand-capped nanoshapes affect the efficacy of chirality transfer to a surrounding reporter medium, here a nematic LC phase, in very predictable ways via independent calculations of 2-D shape compatibility corrected chirality indicators. Each of the gold nanoshapes capped with a chiral ligand shell outperforms the neat organic chiral molecules with respect to their ability to transfer chirality to the N-LC host medium. We established a very systematic correlation between purely geometric concepts and experimental chirality transfer data. This methodology provides a broadly expandable tool to a priori predict experimental chirality transfer efficacy data.

In the described N-LC, this opportunity is realized by measuring the induced helical pitch and calculating the helical twisting power in an induced $\mathrm{N}^{*}$-LC medium for essentially any possible nanoshape varying in size, shape, or AR; all without
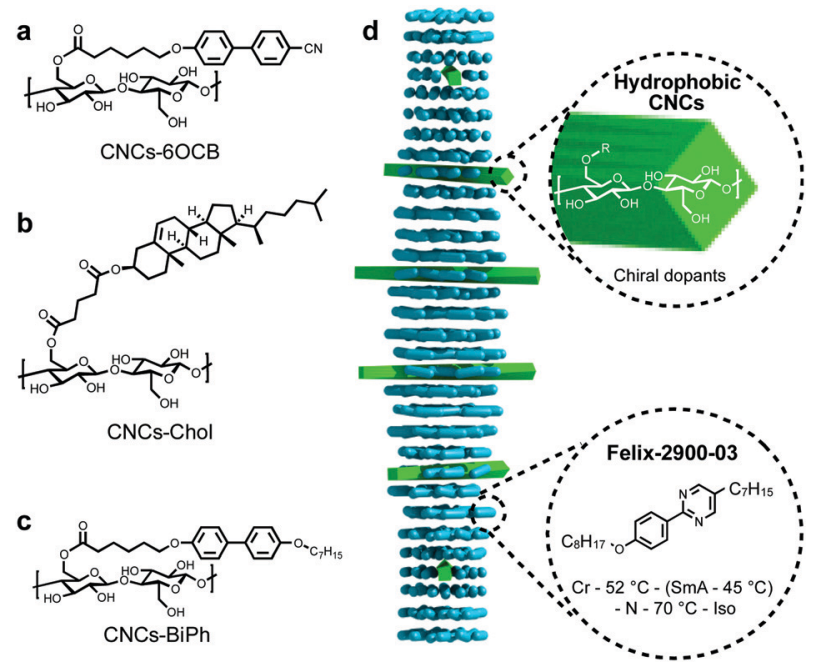

Fig. $10(a-c)$ Chemical structures of the CNC surface modifications. The CNC core displays both morphological (twist of the CNC rods) and inherent core chirality (via the glucose building blocks). (d) Schematic representation of the $\mathrm{N}^{*}$-LC phase induced by the addition of only minute amounts of the functionalized CNCs to the LC host, Felix-2900-03, in the nematic phase. Reproduced from and adapted with permission from ref. 27 - Copyright (2021), Wiley-VCH.

much need to alter the chemical nature of the chiral molecules decorating the nanoshape surface.

But what if the nanoshape core was chiral? To elucidate the role of core chirality, we needed a system with an intrinsically chiral core. The perhaps closest match to the most potent $\mathrm{M}_{\mathrm{AR}}$-GNR1, at least for now, appeared to be cellulose nanocrystals (CNCs). Accordingly, in another recently published study, we further studied such innately chiral CNCs, both neat and decorated with chiral or achiral molecules (Fig. 10). ${ }^{27}$ Here, a comparison between neat CNCs, CNCs-functionalized with cholesterol units (CNCs-Chol), and $\mathrm{M}_{\mathrm{AR}}$-GNR1 revealed that the contribution to chirality transfer from a molecular and morphologically chiral core is likely considerable. The CNCs-Chol, lacking a dense chiral ligand shell present on $\mathrm{M}_{\mathrm{AR}}-\mathrm{GNR} 1$ (translating to a $75 \%$ lower overall cholesterol content for the CNCs-Chol at a set constant weight fraction in an N-LC) showed an about eightfold decrease in $\left|\beta_{\mathrm{w}}\right|$ in comparison to $\mathrm{M}_{\mathrm{AR}}$-GNR1. However, replacing the chiral cholesterol units (Fig. 10b) with achiral pro-mesogenic moieties characterized by a close structural match to the N-LC host molecules (Fig. 10c) did not lead to a reduction of $\left|\beta_{\mathrm{w}}\right|$.

Thus, the dense network of chiral molecules on the surface of the gold nanoshapes is the key driving force for their unusually high ability to transfer chirality to a surrounding achiral N-LC medium, fundamentally affected by shape congruency. Yet, any further enhancement of the chirality transfer efficacy can likely be accomplished by introducing nanoshapes featuring both a chiral core and an $\mathrm{IPR}_{2 \mathrm{D}}$ value matching the one of the N-LC host, which is what current experiments in our labs are focusing on. Such intrinsically chiral cores have been reported for a number of anisometric nanomaterials including, 
for example, in a recent report by Ben-Moshe and colleagues for bipyramidal tellurium $^{28}$ or Markovich et al. for rod-like $\mathrm{Eu}^{3+}$-doped terbium phosphate nanocrystals. ${ }^{29}$ Given the recently increasing number of articles citing shape compatibility arguments, ${ }^{30}$ recognized for simple molecular LC systems by Feringa and co-workers years ago, ${ }^{31}$ we foresee that the utility of the mathematical and computational concepts described here may soon be significantly advanced by machine learning and artificial intelligence (AI) strategies, and as such support familiar 'you cannot put a square peg in a round hole' metaphors for molecular and nanoscale chirality transfer systems. Ultimately, a further expansion of these concepts to lyotropic LC systems ${ }^{32,33}$ that are biologically significantly more relevant as well as to applications seems all but inevitable. ${ }^{34,35}$

\section{Conflicts of interest}

There are no conflicts to declare.

\section{Acknowledgements}

The work by the authors' teams described here was financially supported by the US National Science Foundation (NSF, DMR1506018 and DMR-1904091; TH), the Ohio Third Frontier (OTF) program for Ohio Research Scholars: "Research Cluster on Surfaces in Advanced Materials" (TH), and the Italian MIUR (PRIN project 2015XJA9NT; CZ). The authors also acknowledge Hossein Mirzakhani and Diana P. N. Gonçalves for some of the graphical designs.

\section{Notes and references}

1 L. D. Barron, Chirality, 2012, 24, 879-893; S. M. Morrow, A. J. Bissette and S. P. Fletcher, Nat. Nanotechnol., 2017, 12, 410-419.

2 G. B. Gelmini and M. Roncadelli, Phys. Lett. B, 1981, 99, 411-415; J. Gooth, A. C. Niemann, T. Meng, A. G. Grushin, K. Landsteiner, B. Gotsmann, F. Menges, M. Schmidt, C. Shekar, V. Süß, R. Hühne, B. Rellinghaus, C. Felser, B. Yan and K. Nielsch, Nature, 2017, 547, 324-327.

3 B. A. McGuire, P. B. Carroll, R. A. Loomis, I. A. Finneran, P. R. Jewell, A. J. Remijan and G. A. Blake, Science, 2016, 352, 1449-1452; D. P. Glavin, A. S. Burton, J. E. Elsila, J. C. Aponte and J. P. Dworkin, Chem. Rev., 2020, 120, 4660-4689.

$4 \mathrm{~W}$. T. Kelvin, Baltimore lectures on molecular dynamics and the wave theory of light, C. J. Clay and Sons, London, 1904.

5 M. Inaki, J. Liu and K. Matsuno, Philos. Trans. R. Soc., B., 2016, 371, 20150403; A. S. Chin, K. E. Worley, P. Ray, G. Kaur, J. Fan and L. Q. Wan, Proc. Natl. Acad. Sci. U. S. A., 2018, 115, 12188-12193.

6 W. H. Brooks, W. C. Guida and K. G. Daniel, Curr. Top. Med. Chem., 2001, 11, 760-770.
7 T. P. Yoon and E. N. Jacobsen, Science, 2003, 299, 1691-1693; A. Pfaltz and W. J. Drury III, Proc. Natl. Acad. Sci. U. S. A., 2004, 101, 5723-5726.

8 P. Gutsche, X. Garcia-Santiago, P.-I. Schneider, K. M. McPeak, M. Nieto-Vesperina and S. Burger, Symmetry, 2020, 12, 158.

9 D. Yang, P. F. Duan, L. Zhang and M. H. Liu, Nat. Commun., 2017, 8, 15727.

10 Y. Kim, B. Yeom, O. Arteaga, S. J. Yoo, S.-G. Lee, J.-G. Kim and N. A. Kotov, Nat. Mater., 2016, 15, 461-468.

11 H.-E. Lee, H.-Y. Ahn, J. Mun, Y. Y. Lee, M. Kim, N. H. Cho, K. Chang, W. S. Kim, J. Rho and K. T. Nam, Nature, 2018, 556, 360-365.

12 K. Ariga, T. Mori, T. Kitao and T. Uemura, Adv. Mater., 2020, 32, 1905657.

13 A. B. Buda, T. auf der Heyde and K. Mislow, Angew. Chem., Int. Ed. Engl., 1992, 31, 989-1007.

14 M. Grzelczak, J. Pérez-Juste, P. Mulvaney and L. M. LizMarzán, Chem. Soc. Rev., 2008, 37, 1783-1791.

15 M. A. Osipov, B. T. Pickup and D. A. Dunmur, Mol. Phys., 1995, 84, 1193-1206.

16 A. Pietropaolo, L. Muccioli, R. Berardi and C. Zannoni, Proteins, 2008, 70, 667-677.

17 A. P. Almeida, L. Querciagrossa, P. E. S. Silva, F. Gonçalves, J. P. Canejo, P. L. Almeida, M. H. Goghinho and C. Zannoni, Soft Matter, 2019, 15, 2838-2847.

18 P. G. de Gennes and J. Prost, The Physics of Liquid Crystals, Oxford University Press, Oxford, 1993.

19 A. Sharma, T. Mori, H.-C. Lee, M. Worden, E. Bidwell and T. Hegmann, ACS Nano, 2014, 8, 11966-11976.

20 G. Solladié and R. Zimmermann, Angew. Chem., Int. Ed. Engl., 1984, 23, 348.

21 T. Mori, A. Sharma and T. Hegmann, ACS Nano, 2016, 10, 1552-1564.

22 W. Kuhn, Annu. Rev. Phys. Chem., 1958, 9, 417-438.

23 A. Guerrero, B. Auguié, J. Alonso-Gómez, Z. Džolić, S. GómezGraña, M. M. Cid and L. M. Liz-Mazán, Angew. Chem., Int. Ed., 2011, 50, 5499-5503.

24 A. Nemati, S. Shadpour, L. Querciagrossa, L. Li, T. Mori, M. Gao, C. Zannoni and T. Hegmann, Nat. Commun., 2018, 9, 3908.

25 A. Nemati, S. Shadpour, L. Querciagrossa, T. Mori, C. Zannoni and T. Hegmann, ACS Nano, 2019, 13, 10312-10326.

26 A. Nemati, L. Querciagrossa, C. Callison, S. Shadpour, D. P. N. Gonçalves, T. Mori, X. Cui, R. Ai, J. Wang, C. Zannoni and T. Hegmann, Sci. Adv., 2022, 8, abl4385.

27 D. P. N. Gonçalves and T. Hegmann, Angew. Chem., Int. Ed., 2021, 60, 17344-17349.

28 A. Ben-Moshe, A. da Silva, A. Müller, A. Abu-Odeh, P. Harrison, J. Waelder, F. Niroui, C. Ophus, A. M. Minor, M. Asta, W. Theis, P. Ercius and A. P. Alivisatos, Science, 2021, 372, 729-733.

29 U. Hananel, A. Ben-Moshe, H. Diamant and G. Markovich, Proc. Natl. Acad. Sci. U. S. A., 2019, 116, 11159-11164.

30 M. Weh, J. Rühe, B. Herbert, A.-M. Krause and F. Würthner, Angew. Chem., Int. Ed., 2021, 60, 15323-15327; S.-Y. Zhang, D. Fairen-Jimenez and M. J. Zaworotko, Angew. Chem., Int. 
Ed., 2020, 59, 17600-17606; A. B. George and K. S. Korelev, PLoS Comput. Biol., 2018, 14, e1006645.

31 N. Katsonis, A. Minoia, T. Kudernac, T. Mutai, H. Xu, H. Uji-i, R. Lazzaroni, S. De Feyter and B. Feringa, J. Am. Chem. Soc., 2008, 130, 386-387.

32 S. Shadpour, J. P. Vanegas, A. Nemati and T. Hegmann, ACS Omega, 2019, 4, 1662-1668.
33 L. Bergquist and T. Hegmann, ChemNanoMat, 2017, 3, 863-868.

34 K. Perera, A. Nemati, E. Mann, T. Hegmann and A. Jákli, ACS Appl. Mater. Interfaces, 2021, 13, 4574-4582.

35 D. P. N. Gonçalves, M. E. Prévôt, S. Üstünel, T. Ogolla, A. Nemati, S. Shadpour and T. Hegmann, Liq. Cryst. Rev., 2021, 9, 1-34. 